\title{
Le rôle des techniques de sonde atomique dans l'investigation des transformations de phase
}

\author{
D. BLAVETTE, F. DANOIX et P. AUGER
}

Laboratoire de Microscopie Ionique, URA 808 UFR Sciences, Université de Rouen, 76821 Mont Saint Aignan cedex, France

\begin{abstract}
The role of both Field Ion Microscopy and Atom-Probe techniques in the investigation of solid state phase transformations is discussed on the basis of some examples. The first illustration deals with Long Period Superstructures observed in $\mathrm{Cu}_{3} \mathrm{Pd}$ system. Atom-probe investigations conducted on (001) planes show the occurence of Long Range Order modulations in the vicinity of antiphase boundaries.
\end{abstract}

Because of its very high spatial resolution, atom-probe is also an attractive tool for the study of unmixing processes. Some results related to the spinodal decomposition of the iron-chromium system are discussed in connection with current unmixing theories.

Prospects opened by the emergence of new three-dimensional atom-probes are given.

\section{INTRODUCTION}

Dès son invention par E. W. Müller [1] il y a près de 25 ans, la sonde atomique s'est révélée être une technique de microanalyse de choix pour l'étude des premiers stades de transformation de phase dans les alliages métalliques [2]. Le principe de la sonde atomique et de la microscopie ionique, technique qui lui est toujours associée, est décrit dans une revue détaillée [3]. La résolution spatiale de l'instrument en surface de l'échantillon est voisine du nanomètre, celle en profondeur atteint une distance interatomique. C'est grâce à cette très haute résolution en profondeur que la sonde atomique est un outil séduisant pour l'étude des transformations ordre-désordre. En effectuant l'analyse sur les plans de surstructure d'une phase ordonnée, les sites préférentiels des différents éléments d'addition peuvent être déterminés [5]. De même, il est possible d'accéder aux modulations d'ordre à grande distance existant en particulier près de parois d'antiphase. Nous illustrerons cette possibilité sur la base d'une étude que nous avons menée sur les structures à longues périodes dans les alliages $\mathrm{Cu}_{3} \mathrm{Pd}$ [6]. Bon nombre d'auteurs ont aussi utilisé cet instrument pour mesurer quantitativement la composition des phase en cours de cinétique et en interpréter l'évolution. Dans l'approche classique des processus de séparation de phase, on oppose souvent le phénomène de germination puis croissance d'amas isolés au processus de décomposition spinodale [4]. Dans le premier cas, les premiers germes sont supposés avoir, dès leur formation, une composition proche de la valeur d'équilibre prévue thermodynamiquement. A l'opposé pour le deuxième mode de démixtion, on attend une augmentation graduée de l'amplitude et de la longueur d'onde moyenne des fluctuations de composition jusqu'à l'obtention des concentrations d'équilibre. L'apport majeur de la sonde atomique dans ce cas précis est de permettre d'accéder, dans l'espace direct, à la fois aux amplitudes des fluctuations de concentration et aux longueurs de corrélation. Sur la base des recherches que nous avons menées sur des alliages $\mathrm{Fe}-\mathrm{Cr}$, nous montrerons comment ces informations peuvent être confrontées aux théories actuelles. 


\section{STRUCTURES A LONGUE PERIODE DANS LES ALLIAGES Cu $3 \underline{\text { Pd }}$}

Les structures à longue période sont observées dans bon nombre de systèmes. Elles peuvent se décrire comme l'empilement de cellules $\mathrm{L}_{2}$ séparées par des parois d'antiphase conservatives. Celles-ci sont situées dans les plans (001) de surstructure de la phase ordonnée. Suivant la composition ou la température, les tailles de domaines peuvent être ou non commensurables avec le paramètre cristallin. Dans les alliages $\mathrm{Cu}_{3 \pm x} \mathrm{Pd}$, les images de microscopie électronique en haute résolution révèlent des domaines mono- ou bi-dimensionnels [7]. Différentes topologies peuvent être observées, les parois apparaissant sinueuses ou rectilignes.

Les expériences que nous avons effectuées en collaboration avec l'ONERA [6] portent sur les alliages Cu$18.5 \mathrm{at} \% \mathrm{Pd}$ et $\mathrm{Cu}-20.5 \mathrm{at} \% \mathrm{Pd}$. Les investigations ont été faites sur des monocristaux orientés $<001>$. Une micrographie ionique, correspondant à une projection quasi stéréographique du réseau [3], est présentée figure 1. Seuls les atomes de palladium sont imagés. La micrographie met clairement en évidence la présence d'un réseau de parois d'antiphase parallèles dont l'orientation est perpendiculaire au plan de la photo. Elles apparaissent sous la forme de discontinuités dans les cercles concentriques constituant le pôle (001). La distance moyenne $M$ entre ces parois, estimée à partir du grandissement $\left(\sim 10^{6}\right)$, est tout à fait voisine de la taille moyenne des domaines mesurée en microscopie électronique à haute résolution $(\mathrm{M} \cong$ 10,3 a où $\mathrm{a}$, le paramètre cristallin, vaut $3,7 \AA$ ). La largeur des domaines monodimensionnels est incommensurable avec le paramètre de maille.

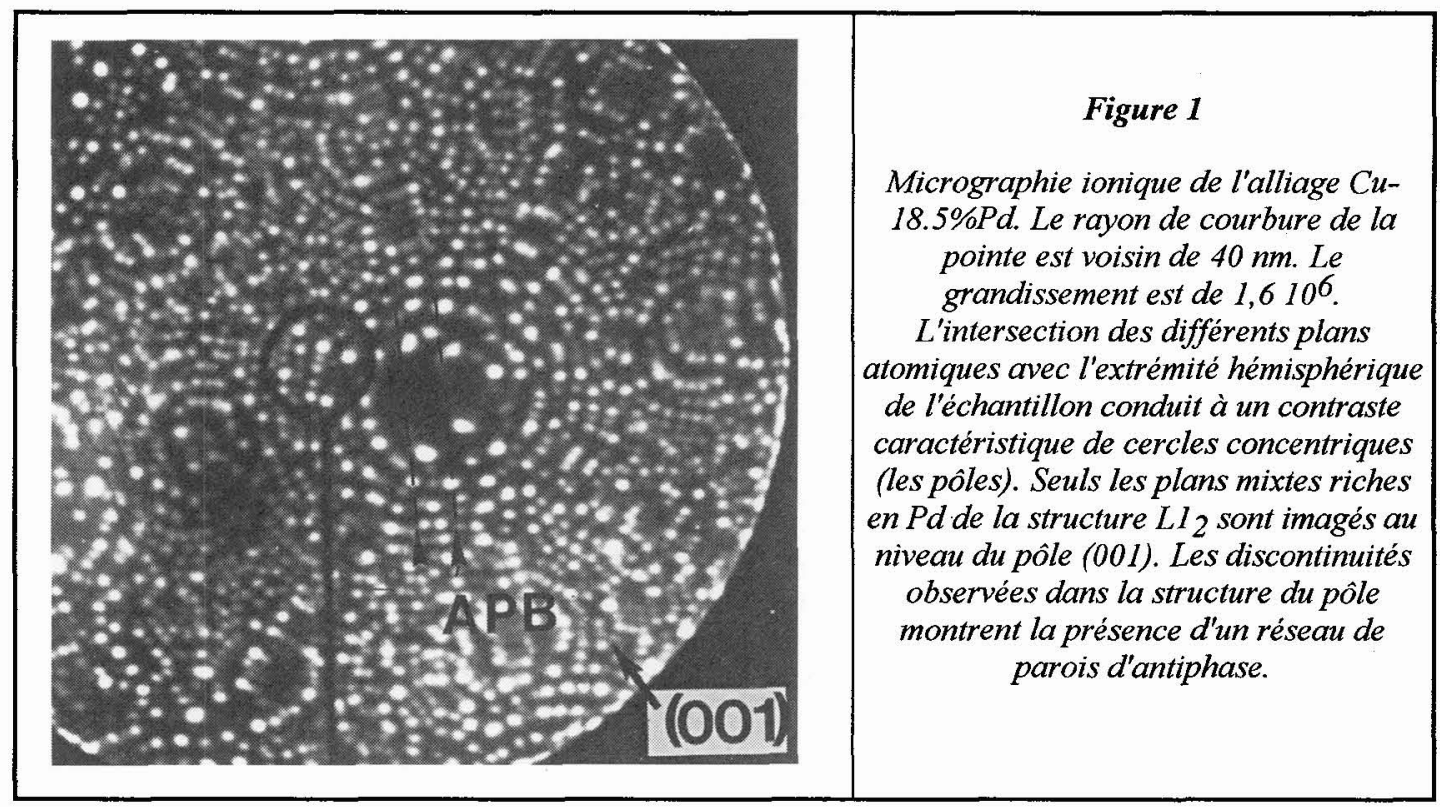

Parmi les trois variantes d'orientations équivalentes dans le structure cubique, seuls les domaines monodimensionnels perpendiculaires à l'axe de la pointe permettent d'accéder aux modulations d'ordre à grande distance. La direction d'analyse, systématiquement parallèle à l'axe de la pointe (001), est alors perpendiculaire aux parois. Un profil de concentration correspondant à l'analyse en profondeur plan atomique par plan atomique de la pointe sur le pôle de surstructure (001) est proposé dans la figure 2. Cet alliage (Cu-20.5\% $\mathrm{Pd}$ ) comporte des domaines dont la largeur, incommensurable avec la paramètre du réseau (a) est égale à $M=6,24$.a [7]. 
Malgré les inévitables fluctuations statistiques d'échantillonnage dues au faible nombre d'ions collectés par plan atomique analysé $(\mathrm{N} \sim 36)$, le profil met clairement en évidence la séquence d'empilement des plans (001) de la structure $\mathrm{Cu}_{3}-\mathrm{Pd}\left(\mathrm{Ll}_{2}\right)$. Il y a alternance de plans mixtes $\mathrm{Cu} / \mathrm{Pd}$ (plans riches en $\mathrm{Pd}$ ) et de plans purs en $\mathrm{Cu}$ (plans pauvres en $\mathrm{Pd}$ ). Cette périodicité de longueur d'onde $\lambda\left(=\mathrm{d}_{001}=\mathrm{a}\right)$ est elle-même modulée : les pics de palladium (correspondant aux plans mixtes) présentent des valeurs extrêmes quasi périodiquement. La distance entre ces maxima maximorum vaut soit 6 soit 7 fois le paramètre cristallin. La longueur moyenne de corrélation est voisine de 6,5.a, ce qui est très proche de la taille des domaines observés en microscopie électronique à haute résolution [6]. Par ailleurs, les caractéristiques de cette modulation de l'ordre à grande distance (observée ici dans l'espace direct) sont en parfait accord avec les clichés de diffraction. L'interprétation des spots satellites qui apparaissent indique que la composition des plans (001) successifs parallèles aux parois doit être modulée avec la même périodicité que les modulations d'antiphase [7].

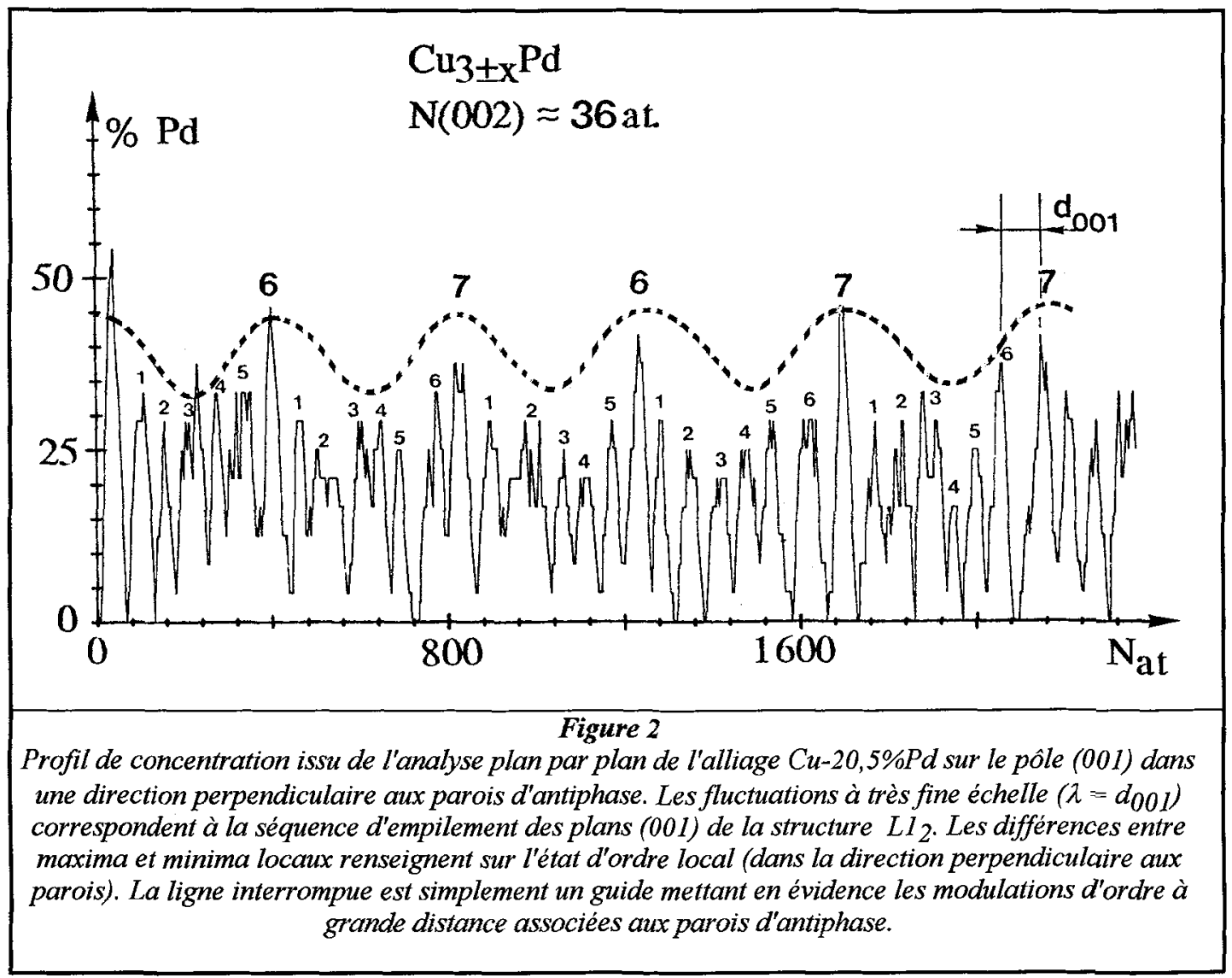

\section{DECOMPOSITION DES PHASES FERRITIOUES Fe-Cr}

L'exemple que nous allons développer maintenant concerne l'étude de la décomposition de la solution solide $\mathrm{Fe}-\mathrm{Cr}$ de la ferrite d'un acier austéno-ferritique en deux phases $\alpha$ et $\alpha^{\prime}$ isomorphes à la phase d'origine et respectivement enrichie en fer et enrichie en chrome. L'acier inoxydable étudié est constitué de deux phases, l'austénite et la ferrite (cf. tableau 1). Dans le domaine choisi de températures $\left(300-400^{\circ} \mathrm{C}\right.$ ), seule la ferrite évolue alors que l'austénite n'est pas affectée [8]. 
Tableau 1 : Compositions chimiques de l'acier inoxydable et de sa phase ferritique déterminées à la microsonde électronique.

\begin{tabular}{|c|c|c|c|c|c|c|c|c|c|c|}
\hline pds \% & $\mathrm{C}$ & $\mathrm{Si}$ & $\mathrm{Mn}$ & $\mathrm{P}$ & $\mathrm{S}$ & $\mathrm{Cr}$ & $\mathrm{Mo}$ & $\mathrm{Ni}$ & $\mathrm{N}$ & Ferrite \\
\hline Acier & 0.039 & 1.22 & 0.60 & 0.028 & 0.010 & 20.0 & 2.56 & 10.0 & 0.027 & $24 \%$ \\
\hline Ferrite & n.d & 1.25 & 0.49 & n.d & n.d & 24.8 & 3.6 & 6.4 & n.d & - \\
\hline
\end{tabular}

n.d.: non déterminé.

Le diagramme d'équilibre $\mathrm{Fe}-\mathrm{Cr}$ présente une lacune de miscibilité étendue. Pour cet acier, dans les conditions de compositions et de traitements thermiques choisies, la solution solide ferritique obtenue après trempe est représentée par un point situé profondément à l'intérieur de la lacune de miscibilité, dans une région où cette solution solide est instable vis à vis de petites fluctuations de concentration. Au cours, d'un vieillissement isotherme ultérieur, l'amplitude de ces fluctuations peut croître continûment, en principe jusqu'à l'obtention des compositions d'équilibre. C'est le phénomène de décomposition spinodale.

La figure 3 présente une micrographie ionique de la ferrite décomposée. Une succession de ces images ioniques obtenues par évaporation des atomes à la surface de la pointe, couche atomique après couche atomique, montre que la microstructure obtenue est isotrope. Elle est formée des deux labyrinthes des domaines $\alpha$ et $\alpha^{\prime}$ interconnectés comme le sont les deux constituants d'une éponge.

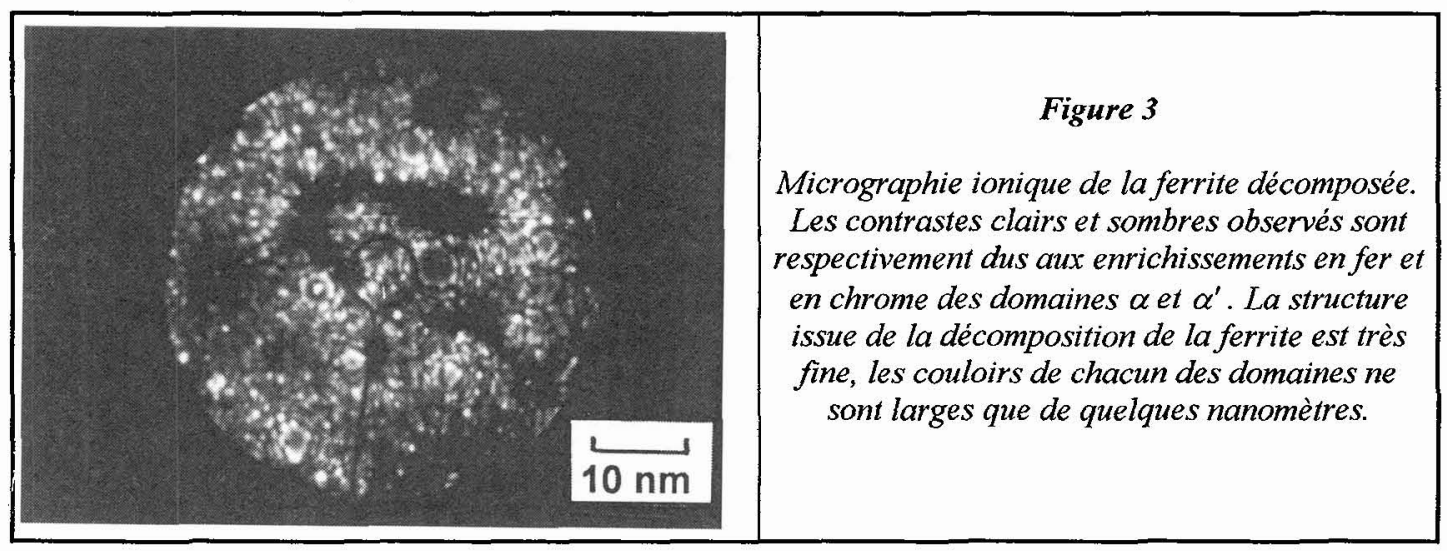

Comme la structure est isotrope, toutes les directions d'analyse sont équivalentes. La figure 4 montre, en fonction des traitements thermiques, l'évolution des profils de concentration du chrome, élément utilisé comme "traceur" du phénomène étudié.

La cinétique de démixtion apparaît clairement caractérisée par un accroissement continu, au cours du vieillissement, de l'amplitude et des longueurs d'onde des fluctuations de concentration. La détermination des longueurs de corrélation entre domaines enrichis en chrome peut se faire à l'aide d'outils statistiques tels que la transformation de Fourier et l'autocorrelation. Conformément aux prévisions de la théorie classique de la décomposition spinodale de Langer, Bar On et Miller (LBM) [9] et en accord avec les résultats obtenus en diffusion des neutrons pour cet alliage[10], ainsi que sur des binaires fer-chrome (voir par exemple [11-12]), il est possible de mettre en évidence une "longueur d'onde" principale qui suit une loi cinétique en $\mathrm{t}^{\mathrm{n}}$ avec, pour l'exposant $\mathrm{n}$, une valeur proche de 0,2 [13].

Pour quantifier l'évolution des amplitudes de fluctuation de la concentration en chrome, nous avons développé une méthode originale qui se fonde sur l'analyse des distributions de fréquences d'observation des concentrations (cf. figure 4a). Chaque distribution, pour un traitement thermique donné, est caractérisée par le paramètre $\mathrm{V}(\mathrm{t}, \mathrm{T})$ égal à la distance statistique qui sépare cette distribution expérimentale de la distribution aléatoire de Bernouilli représentant l'état obtenu juste après trempe [14]. 
L'augmentation des amplitudes de fluctuation se traduit par un accroissement de $\mathrm{V}$ qui est donc une mesure du degré d'avancement de la démixtion. L'évolution de ce paramètre suit une loi de type Arrhénius [13] indiquant que le processus de vieillissement est thermiquement activé et qu'il est le même pour l'ensemble des traitements thermiques étudiés. La valeur unique de l'énergie d'activation $Q$ mesurée est égale à celle de la diffusion du chrome dans le fer $\left(\sim 230 \mathrm{~kJ}^{\mathrm{mol}}{ }^{-1}\right)$, confirmant que c'est bien ce dernier processus qui contrôle la décomposition.

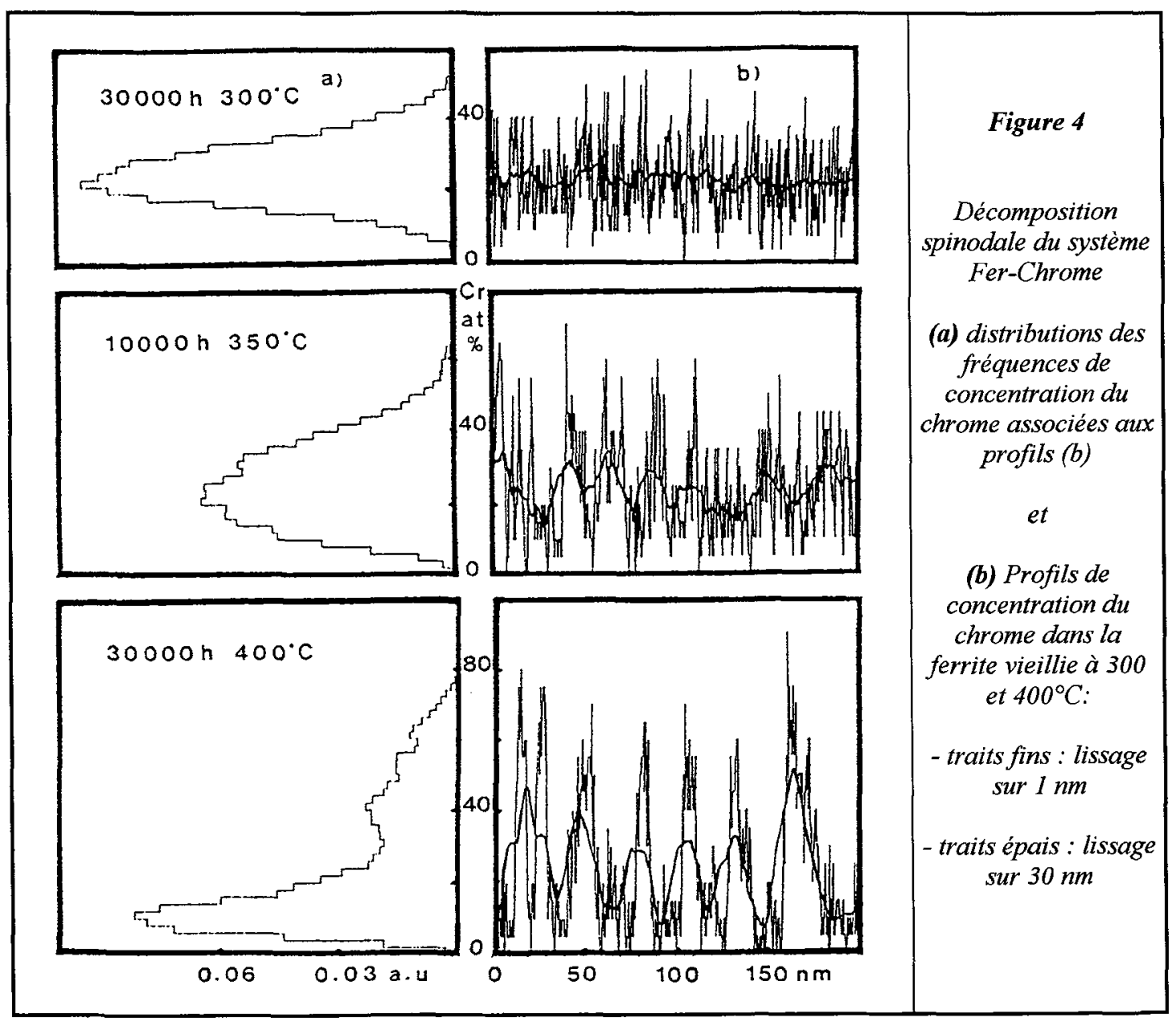

Afin de tester la pertinence des hypothèses avancées par les théories de la décomposition spinodale de Cahn [14] d'une part et de Langer et al. d'autre part, nous avons confronté les distributions de fréquences de concentration calculées à partir de ces dernières avec celles obtenues expérimentalement [15]. Il apparaît ainsi que le modèle linéaire de Cahn ne peut décrire que les tout premiers stades de démixtion tant que les fluctuations restent de faibles amplitudes et symétriques par rapport à la composition moyenne. Lorsque cette condition n'est plus remplie, l'asymétrie observée peut être décrite avec succès à partir de l'hypothèse d'une distribution bigaussienne empruntée au modèle LBM. Ce dernier permettant aussi de rendre compte des premiers stades de séparation des phases, il apparait de portée plus générale que celui de Cahn, bien qu'il ne puisse, lui non plus, interpréter correctement la coalescence de la structure $\alpha-\alpha$ '.

Ce dernier stade de la démixtion se caractérise par une réelle complexification des profils de concentration non prévue par les théories (cf. figure $4 \mathrm{~b}$ ). Contrairement aux schémas classiques, cette étape débute alors 
que la décomposition spinodale n'a pas encore formé les phases d'équilibre. Plus exactement, la phase $\alpha$ appauvrie en chrome atteint "normalement" sa composition d'équilibre $(\sim 10 \mathrm{at} \%)$. En revanche, la phase $\alpha^{\prime}$ n'atteint sa concentration d'équilibre $(\sim 80 \mathrm{at} \%)$, si toutefois elle l'atteint, que dans des régions de très faible extension spatiale ( quelques $\mathrm{nm}$ ) qui représentent une très petite fraction volumique. Cette curiosité est assez bien mise en évidence par les histogrammes de distribution de fréquences qui présentent un étalement des valeurs de la concentration en chrome jusqu'à 80 at \% (figure 4a). Il apparaît par ailleurs, un pic quasistationnaire autour de $40 \mathrm{at} \%$, comme si les domaines $\alpha^{\prime}$ ayant cette composition avaient une certaine métastabilité et se comportaient en structure transitoire.

A ce stade de la coalescence, ces particularités concernant l'évolution des amplitudes sont à relier à l'apparition de fluctuations de concentration de chrome à grandes distances $(\sim 100 \mathrm{~nm})$ qui se superposent aux fluctuations déjà observées ( 10 à $20 \mathrm{~nm}$ ). L'amplitude et la longueur d'onde de ces fluctuations à grandes distances croissent également avec le vieillissement. Elles modulent la concentration moyenne locale de la ferrite, modulant également ainsi la force motrice de décomposition. Celle-ci s'amplifie lorsque la concentration locale en chrome s'élève ce qui renforce ainsi les oscillations. Malgré ce mécanisme, ce qui caractérise la cinétique de cette coalescence, c'est sa grande lenteur. Le stade final n'est toujours pas atteint après 30000 heures à $400^{\circ} \mathrm{C}$ comme en témoigne les mesures de la dureté qui continue de croître.

\section{CONCLUSION}

Aux cotés des techniques plus classiques telles la microscopie électronique ou la diffusion des neutrons, la sonde atomique est un outil tout à fait précieux. Elle permet d'accéder à toutes sortes d'informations originales et complémentaires, et ce, à une échelle extrêmement fine.

Tout récemment, une nouvelle génération de sonde atomique permettant l'obtention d'images quantitatives tridimensionnelles est apparue [18]. L'appareil que nous avons conçu et mis au point, permet la reconstruction, atome par atome, d'un petit volume de matériau à une échelle proche de la maille cristalline. Chaque atome est parfaitement identifié par sa masse. De véritables cartographie des hétérogénéités chimiques résolues à une échelle subnanométrique sont ainsi obtenues. Avec l'émergence de cet instrument, c'est une nouvelle approche des mécanismes de transformation de phase qui débute.

\section{Bibliographie}

[1] MULLER E. W., PANITZ J. A. and Mc LANE S. B., Rev. Sci. Instr. 39 (1968) 83-86

[2] MILLER M. K. and SMITH G. D. W., Atom-Probe microanalysis : Principle and applications to materials problems, Mat. Res. Soc. (1989) Pittsburgh, Pa.

[3] BLAVETTE D. et MENAND A., Annales de chimie, 11 ( 1986) 321-384

[4] MARTIN G., Ecole d'été de Métallurgie d'Aussois, Edit. đe Phys. (1987) 337

[5] BLAVETTE D. et MENAND A., C. R. A. S, Serie II, t 298, 20 (1984) 865-870

[6] BLAVETTE D., CHAMBRELAND S., LOISEAU A., PLANES J. and DUCASTELLE F., J. de Phys. C8-50 (1989) 365-370

[7] BRODDIN D., VAN TENDELOO G., VAN LANDUYT J., AMELINCKX S., PORTIER R., GUYMONT M. et LOISEAU A., Phil. Mag. A. 54 (1986) 395-419

[8] AUGER P., DANOIX F., MENAND A., BONNET S., BOURGOIN J., GUTTMANN M.

Mat. Sci. Technol., 6, (1990) 301

[9] LANGER J.S., BAR ON M., MILLER H.D., Phys. Rev. A., 11 (1975) 1417

[10] MASSOUD J.P., AUGER P., DANOIX F., REZAKHANLOU R., VAN DUYSEN J.C., Actes : 6th International Symposium on Environmental Degradations in Nuclear Power Systems Water Reactors, San Diego, Août 1993. A paraître

[11] LaSALLE J.C., SCHWARTZ L.H., Acta. Met, $\underline{34}$ (1986) 989

[12] BLEY F., Acta. Met, 40 (1992) 1505

[13] DANOIX F., AUGER P., BLAVETTE D., Surf. Sci., 266 (1992) 364-369

[14] AUGER P., MENAND A., BLAVETTE D.,J. de Phys. 49, 66 (1988) 439

[15] AUGER P., DANOIX F., GUTTMANN M., BLAVETTE D.

"Duplex Stainless Steels'91" Ed. J. CHARLES and S. BENHARDSSON, Les Editions de Physique, 1 (1991) 111

[16] CAHN J.W., Trans. AIME., 242 (1968) 166

[17] DANOIX F., DECONIHOUT B., BOS.TEL A., AUGER P., Surf. Sci., 266 (1992) 409

[18] BLAVETTE D., BOSTEL A., SARRAU J.M., DECONIHOUT B., and MENAND A., Nature 363 (1993) 432-435 